\title{
Analysis of Breast Thermography Using Fractal Dimension to Establish Possible Difference between Malignant and Benign Patterns
}

\author{
Mahnaz EtehadTavakol ${ }^{1,5}$, Caro Lucas ${ }^{2}$, Saeed Sadri ${ }^{3,5}$ and E.Y. K. $\mathbf{N g}^{4}$ \\ ${ }^{1}$ Electrical and Computer Engineering Department, \\ Isfahan University of Technology, Iran \\ m.etehadtavakol@ec.iut.ac.ir \\ ${ }^{2}$ Electrical and Computer Engineering Department, \\ University of Tehran, Tehran, Iran \\ lucas@ipm.ir \\ ${ }^{3}$ Electrical and Computer Engineering Department, \\ Isfahan University of Technology, Iran \\ sadri@cc.iut.ac.ir \\ ${ }^{4}$ School of Mechanical and Aerospace Engineering, College of Engineering, \\ Nanyang Technological University, 50 Nanyang Avenue, Singapore 639798 \\ mykng@ntu.edu.sg \\ ${ }^{5}$ Medical Image and Signal Processing Research Center, \\ Isfahan University of Medical Science, Isfahan 81746-73461, Iran \\ kolahdouzan@mail.mui.ac.ir \\ corresponding author: $E Y K N g$, \\ mykng@ntu.edu.sg
}

\begin{abstract}
Early detection of breast cancer by means of thermal imaging has a long and extremely controversial history. Recently, the availability of highly sensitive infrared (IR) cameras which can produce high-resolution diagnostic images of the temperature and vascular changes of breasts, as well as a better knowledge of advanced image processing techniques, has generated a renewed interest. The objective of this study is to investigate fractal analysis of breast thermal images and to develop an algorithm for detecting benignity and malignancy of breast diseases. The study is based on IR images captured by thermal camera, in which the resolution of the results is within the state of the art of IR camera. A total of 7 malignant cases and 8 benign cases have been considered. The breast images were first segmented by fuzzy c-means clustering. Then the first hottest regions for each image were identified and the fractal dimension of those regions was computed. It is shown that the fractal dimension results significantly differ between malignant and benign patterns, suggesting that fractal analysis may potentially improve the reliability of thermography in breast tumor detection.
\end{abstract}

Keywords: Angiogenesis; Fractal analysis; Breast; Tumor shapes; Thermography; Fuzzy 


\section{INTRODUCTION}

Breast diseases are one of the major issues in women's health today. Early detection of breast cancer plays a significant role in reducing the mortality rate. By identifying and removing malignant tumors in early stages before they metastasize and spread to neighboring regions, cancer threats could be halted.

Advances in infrared (IR) camera technologies and properly calibrated thermography have lead to an increased interest in the application of IR system in the medical fields especially in breast cancer detection. Breast thermography with proper protocols [1] is a potential early detection method which is non-invasive, non-radiating, passive, fast, painless, low cost, risk free with zero contact with the body $[2,3,4,5,6]$. Breast thermography is suitable for women in all ages, including pregnant or nursing women, with all sizes of breast, with or without breast implants, fibrocystic breasts, and breast of dense tissue [7]. Blood vessel activity and heat indicate the presence of precancerous cells or cancer cells in their early stages of development. It can detect the early sign of cancer 8 to 10 years before mammography does [8]. While in functional thermography, physiological changes are captured, in mammography, anatomical changes are detected. It is worth noting that physiological changes eventually would lead to anatomical changes.

Cancer is often characterized as a chaotic, poorly regulated growth [9]. Cancerous cells, tumors, and vasculature defy have irregular shapes which can not be described by traditional Euclidean geometry based on smooth shapes such as line, plane, cylinder, and sphere. Fractal geometry is thus useful when the focus is on irregularities of tumor growth. Fractal geometry is a more powerful means of quantifying the spatial complexity of real objects [10].

This paper is organized as follows: Factors that determine breast temperature are explained in section 2. Fractals in Biomedical Images are presented in section 3. Fractal dimension (FD) is discussed in section 4. Section 5 analyzes dataset, describes steps of the processing, and presents the results obtained for 3 benign and 3 malignant selected typical test cases. Section 6 concludes the findings.

\section{FACTORS THAT DETERMINE BREAST TEMPERATURE}

\subsection{Angiogenesis}

There have been a number of studies on the angiogenesis of tumors. The vascular architecture of tumors is known to be significantly different from that of normal tissues [11]. Tree like branching patterns or nearly constant intravascular distances are consistent features in normal vascular networks, while no such features exist in disorganized tumor vascular networks. There are some normal situations that angiogenesis occurs. For example, in wound repairing, the fundamental process of the development and growth of new blood vessels from the pre-existing vasculature is highly regulated. Another example of normal angiogenesis is in a developing child in mother's womb where the vast network of arteries, veins, and capillaries are created [12].

Some investigations have been conducted with regard to angiogenesis in breast thermography. In 1996, Gamagami observed hypervascularity and hyperthermia in $86 \%$ of non-palpable breast cancers with thermograms [13]. He also noted that in $15 \%$ of those cases, thermography helped to detect cancers that were not visible through mam- 
mography. Guido and Schnitt proposed that angiogenesis is an early event in the development of breast cancer and it might occur before tumor cells acquired the ability to invade the surrounding stoma and even before there was any morphologic evidence of a ductal carcinoma in situ (DCIS) [14]. Accordingly, angiogenesis has been identified as a critical event in tumor growth. The influences of blood flow and red blood cell heterogeneity on tumor growth and angiogenesis have been investigated by Alarcon et al. with a mathematical model [15]. Levine et al. introduced another model of tumor angiogenesis taking into consideration biochemical processes [16].

\subsection{High Metabolic Rate Angiogenesis}

Tumor angiogenesis involves proliferation of a network of blood vessels which supply nutrients and oxygen into tumor cells and remove waste products from them [17]. Cancerous tumor cells release molecules that send signals to surrounding normal host tissue. By these signals, certain gens (oncogenes) in the host tissue could be activated and make proteins to encourage growth of new blood vessels.

Nitroxide distribution is different between the normal and tumor tissues. The differences in vasculatures of microenviroment associated with tumors may be reflected by this fact. Also, the nitroxide distribution differs substantially in tumors of different sizes [18]. Scalerandi et al. modeled the local interaction between cells and the vascular system [19].

\subsection{Local Vasodilatation}

Anbar $[20,21]$ suggested that some observations indicated that enhanced perfusion over a substantial area of breast surface via tumor-induced nitric oxide vasodilatation in small tumor could be capable of producing notable IR changes.

\section{APPLIATIONS OF FRACTAL ANALYSIS IN BIOMEDICAL IMAGES}

Fractal analysis has been widely applied in biomedical areas. In a study on the irregularity of the borders of meloanocytic lesions, Lee et al. compared several shape factors, including fractal dimension (FD) [22]. Using fractals, abnormal regions in mammograms were identified in a model proposed by Zheng and Chan [23]. Guo et al. used FD in mammograms to detect abnormality of breast masses by support vector machine [24]. Caldwell et al. [25] and Byng et al. [26] computed FD by box counting method to characterize the breast tissue. The fractal concept was employed by Gazit et al. to analyze the vessel networks that surround a tumor and the hemodynamics within these vessel structures [27].The surface FD proposed by Grizzi et al. was able to explain the geometric complexity of cancerous vascular networks [28]. They showed that the number of vessels and their patterns of distribution had a significant impact on the surface FD.

Works were also conducted in Magnetic Resonance Images of brain [29,30] ultrasonic images of liver [31] and images related to colonic cancer [32] using FD for the classification of tumors. Rangarraj et al. employed fractal analysis for the classification of breast masses by using only their contours [33].

Fractal analysis plays an important role in distinguishing malignant tumors from benign tumors in mammograms [34]. Several studies have been proposed relating the edge sharpness of malignant mass as well as the benign mass. Benign masses are usu- 
ally round and smooth with well defined boundaries, while malignant masses are rough and have complicated boundaries [35,36]. Geometrical complexity can be evaluated by quantifying the irregularities of the boundary.

\section{FRACTAL DIMENSION (FD)}

A fractal is a non-regular geometric shape that can be split into parts which posses self similarity or have the same degree of irregularity on all scales [37-43]. In a fractal geometry, the FD is a statistical quantity that indicates how completely a fractal would fill the space in different scales or magnification. Mandelbrot introduced the concept of fractal to denote an object whose Hausdorff dimension is greater than its topological dimension [37].

The following power law describes the relation between $D$, the self similarity dimension, and $a$, the number of self similar pieces at reduction factor $(1 / S)$ :

$$
a \propto \frac{1}{S^{D}}
$$

where $\mathrm{D}$ is defined as:

$$
D=\frac{\log (a)}{\log \left(\frac{1}{S}\right)}
$$

D can be estimated by slope of the straight line approximation for a plot of $\log (a)$ vs. $\log (1 / \mathrm{s})$. There are several approaches in estimating FD, such as analytical and box counting methods. It is possible to generate fractals using an analytical rule which is based upon a recursive mathematical relation. Box counting method (BCM) can be described as partitioning the image into square boxes with equal sizes and then counting the number of boxes which contain a part of the image. The process is repeated with partitioning the images into smaller and smaller size of boxes. The plot of logarithm of the number of boxes counted vs. the logarithm of the magnification index for each stage of partitioning would help in computing the FD of pattern. The slope of best fitting line to the aforementioned plot is the FD of pattern.

To illustrate BCM, a grid of boxes is laid over the curve as shown in Figure 1. The number of grid boxes that contain a part of the curve (viz. the boxes having intersections with the curve, shaded in gray) is calculated. In Fig. 1, the number of these boxes is 10 out of 25 boxes, thus $a=10,1 / S=25$. This is continued for increased numbers of squares, and the FD is given by the gradient of the logarithm of the number of squares occupied by the edge contour, viz. $\log (a)$, over the logarithm of the number of squares, $\log (1 / \mathrm{s})$.

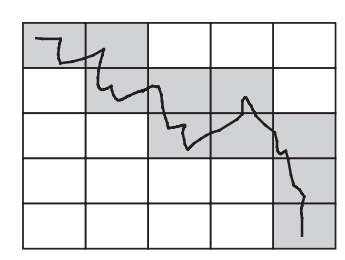

Figure 1. Box counting method 
Figure 2 shows ten sample contours of different irregularities. Their FDs calculated by BCM are exhibited in Table 1, demonstrating that a more complicated contour yields a larger value of FD.

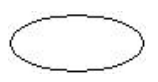

(1)

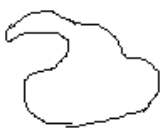

(6)

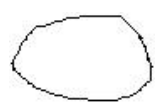

(2)

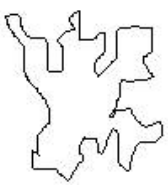

(7)

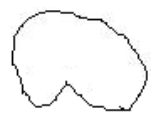

(3)

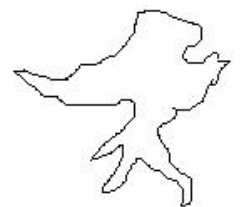

(8)

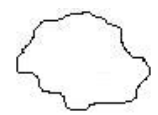

(4)

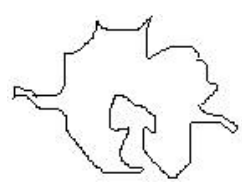

(9)

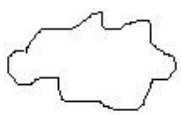

(5)

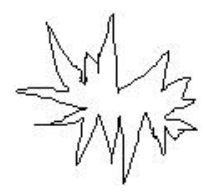

(10)

Figure 2. Sample contours of different irregularities

Table 1. Calculated FD values for the sample contours in Figure 2

\begin{tabular}{lcccccccccc}
\hline Contours & $\mathbf{1}$ & $\mathbf{2}$ & $\mathbf{3}$ & $\mathbf{4}$ & $\mathbf{5}$ & $\mathbf{6}$ & $\mathbf{7}$ & $\mathbf{8}$ & $\mathbf{9}$ & $\mathbf{1 0}$ \\
\hline FD & 1.0711 & 1.1573 & 1.2162 & 1.2204 & 1.2750 & 1.2962 & 1.4076 & 1.4194 & 1.4370 & 1.4464
\end{tabular}

It is demonstrated above that FD can be a potentially useful tool for describing the pathological architecture of breast tumors and providing insights into the mechanisms of tumor growth. In this work, we investigate whether the vascular networks in thermal images posses a fractal nature and if so, what would be the FD values in different stages of the disease.

\section{DATASETS AND PROCESSING STEPS AND RESULTS:}

We studied 15 breast thermograms available from Ann Arbor thermography center [44], Thermal imaging lab in the San Fransisco Bay Area [45], American College of Clinical Thermology [46], Thermography of Iowa [47], and Sunstate Thermal Imaging Center in Australia [48]. Although the images from these sources are varied in their resolutions and generally did not follow a unified protocol, our fractal analysis could demonstrate significant difference between the benign and malignant cases.

One way to segment different colors in breast thermal images is by fuzzy c-means [49] with no empty cluster. Since the fuzzy clustering algorithm compares the colors in a relative way and groups them in clusters which are not with crisp boundaries, each data point can belong to more than one cluster.

In this work, the left breast was separated from the right breast automatically. It included extraction of left and right body boundaries by using Canny edge detector and two morphology operators. Extraction of the two lower boundaries of the breasts was 
achieved as follows: For a data set, a training procedure localized nine landmark points for two breasts. The first and the last points were corresponding to the points with maximum curvature of two breasts. Then for a new case, the two points with maximum curvature of breasts were determined and geometrically transformed to the first and the last points of the averaged set points of the training results. Next, a linear interpolation was applied to fit two curves between the points with maximum curvature of each breast and the fifth point of it. It worked perfectly for $90 \%$ of all the cases at hand.

The following algorithm is proposed for detecting malignancy or benignity of breast tumors:

1) The breast IR images are segmented by fuzzy c-means algorithm [49]. The number of clusters depends on the number of the camera palette colors.

2) The first hottest regions are identified based on the color related to the maximal temperature. The axilla and close sternal boundaries are removed and are not considered.

3) The FD of step (2) is calculated with BCMs as follows:

- $\quad$ Making the image of the first hottest regions in binary.

- Detecting the edges.

- $\quad$ Setting up a grid of boxes.

- Counting the number of occupied boxes.

- Changing the boxes sizes and repeating the previous step.

- Calculating the slope of the best fitting line to the plot of the log of the number of box count vs. the log of the number of boxes.

The box-counting fractal dimension is sensitive to the scale range. This problem is resolved by normalizing the images before using box counting method.

In this study, 8 benign cases and 7 malignant cases were studied following the above algorithm. Three typical benign cases of breast thermograms are shown in Figs. 3a, 4a, and 5a, respectively. Segmentation of their images implemented using fuzzy c-mean is illustrated respectively in Figs. $3 b, 4 b$, and 5 b. The first hottest regions of thermal images are identified in Figs. $3 c$, $4 c$, and $5 c$, with the axilla and close sternal boundaries removed in Figs. 3d1, 4d1, and 5d1, and their corresponding boundaries depicted in Figs. 3d-2, 4d-2, and 5d-2. Plot of the number of box count $(\mathrm{BC})$ vs. the number of boxes $(\mathrm{NB})$ and plot of the $\log (\mathrm{BC})$ vs. $\log (\mathrm{NB})$ are presented in Figs. 3e, 4e, and 5e, respectively. Similar information for the three typical malignant cases is presented in Figs. 6, 7, and 8, respectively. 


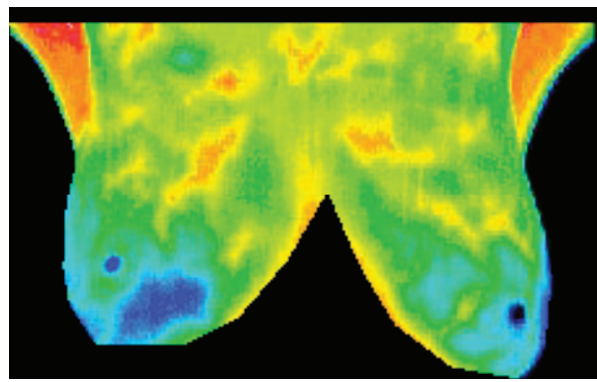

Figure 3a. Benign case 1 (B1).
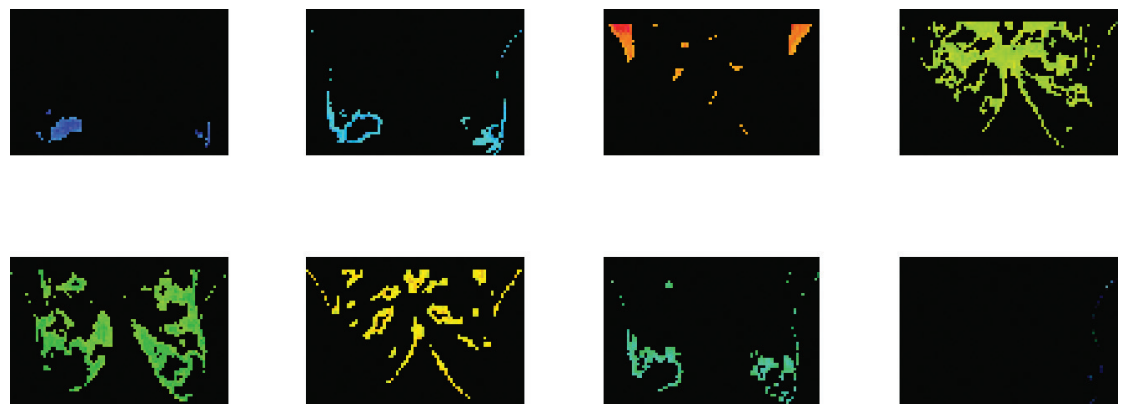

Figure 3b. Segmentation of Figure 3a by fuzzy c-mean.

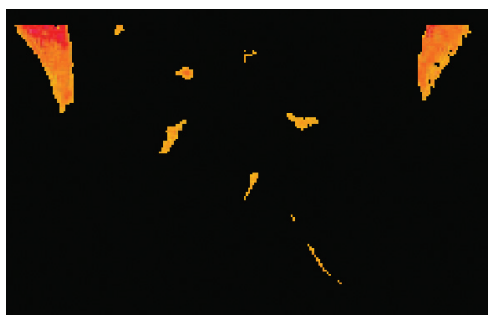

Figure 3c. The first hottest regions.

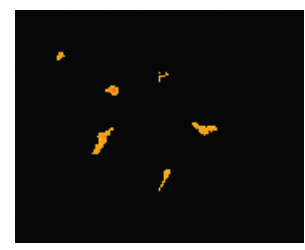

(1)

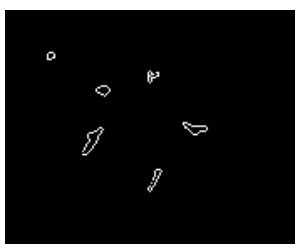

(2)

Figure 3d. (1) The first hottest regions after the axilla and close sternal boundaries are removed.

(2) Boundary contours of (1). 


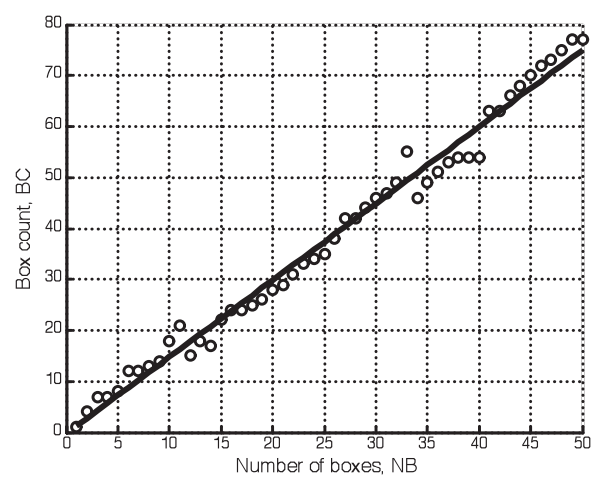

(1)

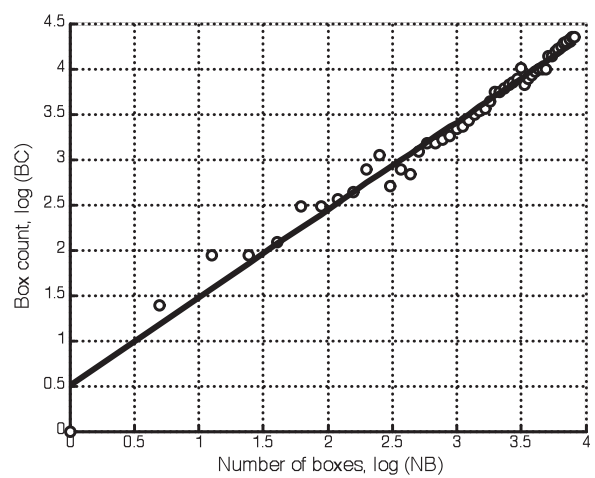

(2)

Figure 3e. (1) Box count (BC) vs. number of boxes (NB). (2) $\log (\mathrm{BC})$ vs. $\log (\mathrm{NB})$.

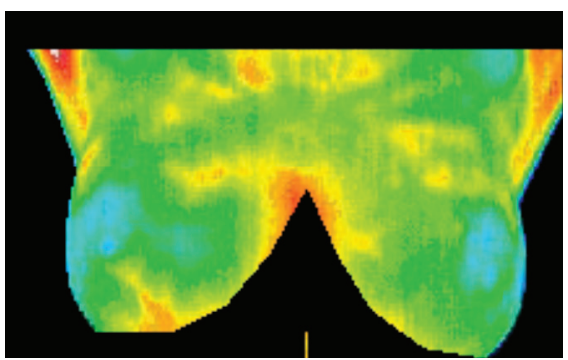

Figure 4a. Benign case 2 (B2).
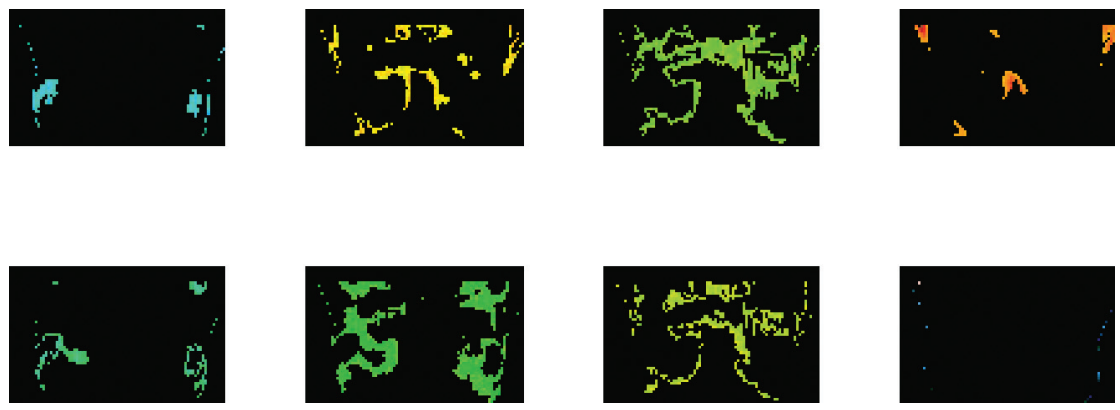

Figure 4 b. Segmentation of Figure 4 a by fuzzy c-mean.

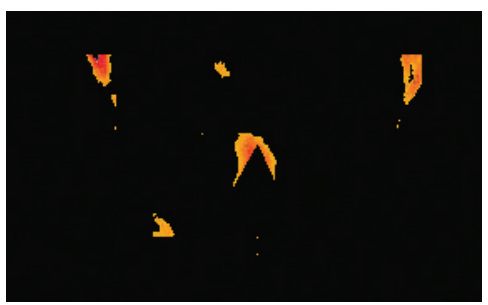

Figure 4c. The first hottest regions. 


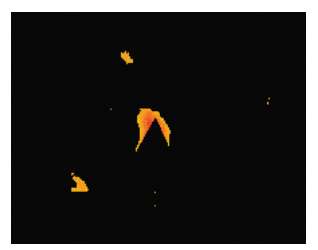

(1)

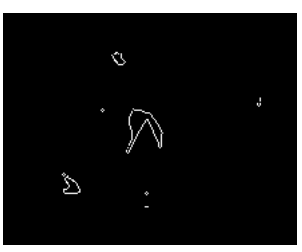

(2)

Figure 4d. (1). The first hottest regions after the axilla boundaries are removed.

(2) Boundary contours of part (1).

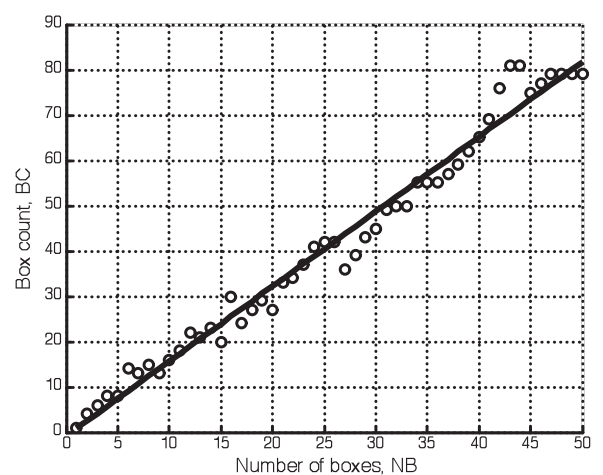

(1)

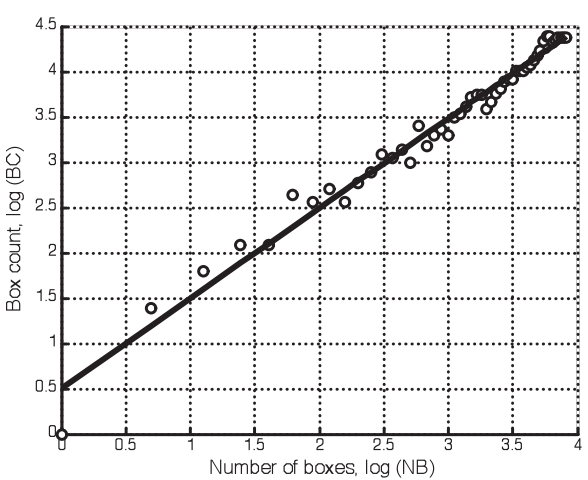

(2)

Figure 4e. (1) Box count (BC) vs. number of boxes (NB). (2) $\log (\mathrm{BC})$ vs. $\log (\mathrm{NB})$.

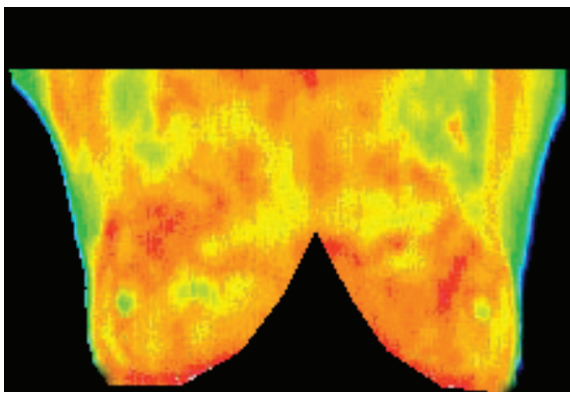

Figure 5a. Benign case 3 (B3).
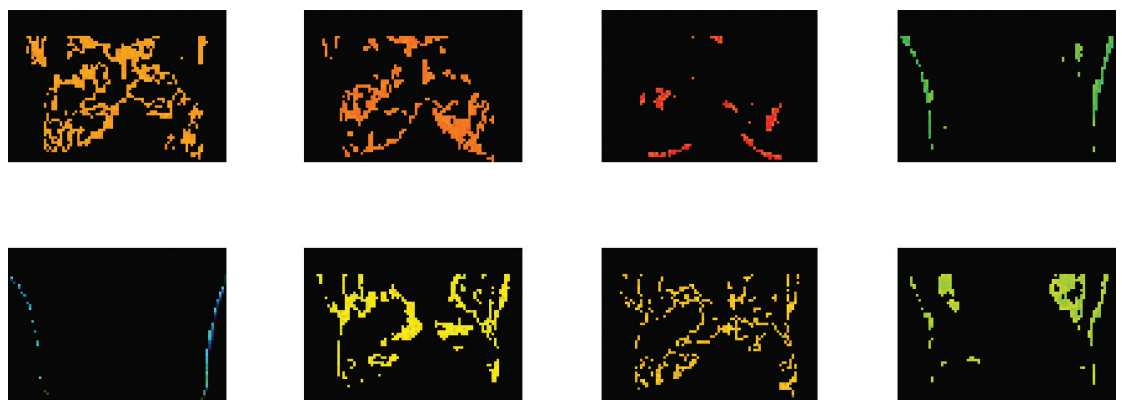

Figure 5b. Segmentation of Figure 5a by fuzzy c-mean. 


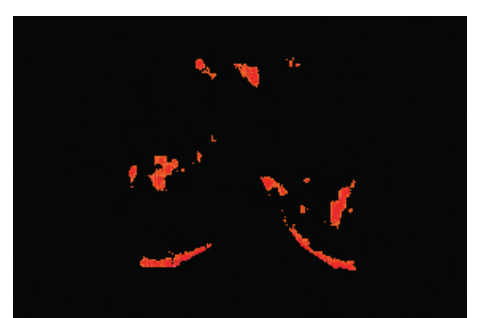

Figure 5c. The first hottest regions.

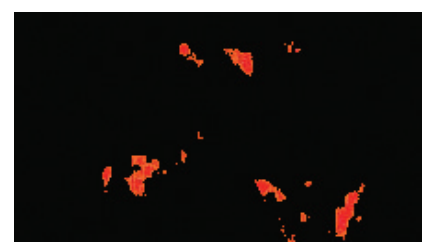

(1)

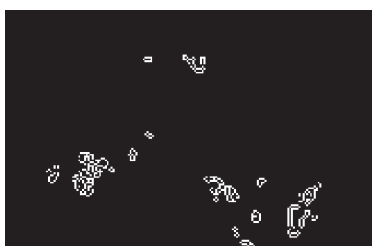

(2)

Figure 5d. (1) The first hottest regions after close sternal boundaries are removed. (2) Boundary contours of part (1).

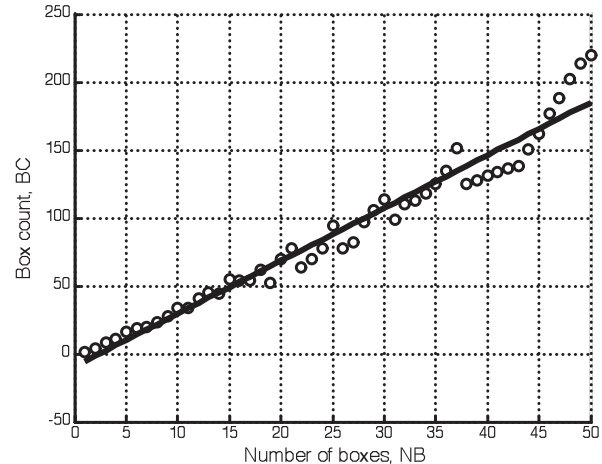

(1)

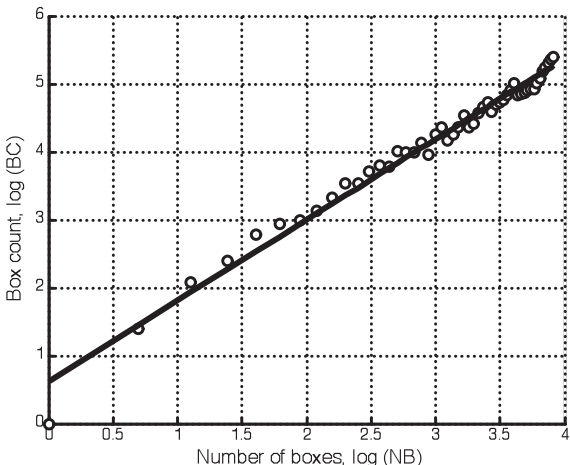

(2)

Figure 5e. (1) Box count (BC) vs. number of boxes (NB). (2) $\log (\mathrm{BC})$ vs. $\log (\mathrm{NB})$.

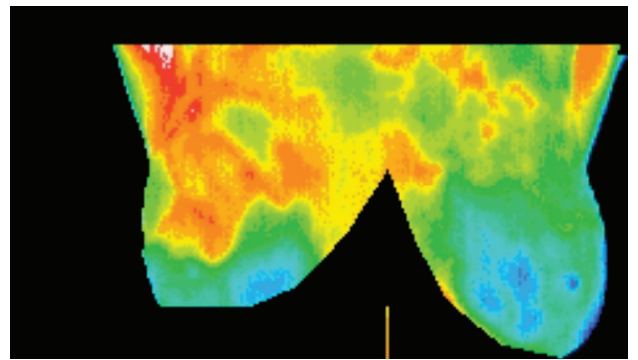

Figure 6a. Malignant case 1 (M1). 

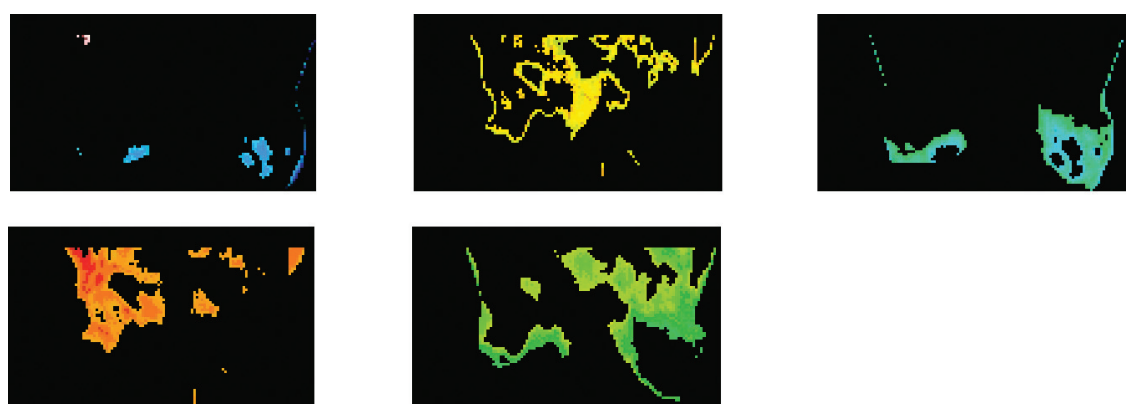

Figure 6b. Segmentation of Figure 6a by fuzzy c-mean.

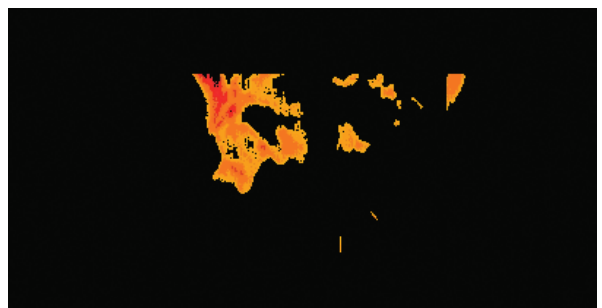

Figure 6c. The first hottest regions.

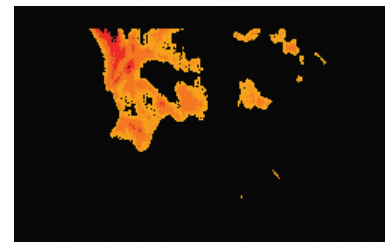

(1)

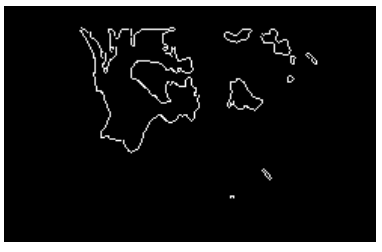

(2)

Figure 6d. (1) The first hottest regions after the axilla boundaries are removed. (2) Boundary contours of part (1).

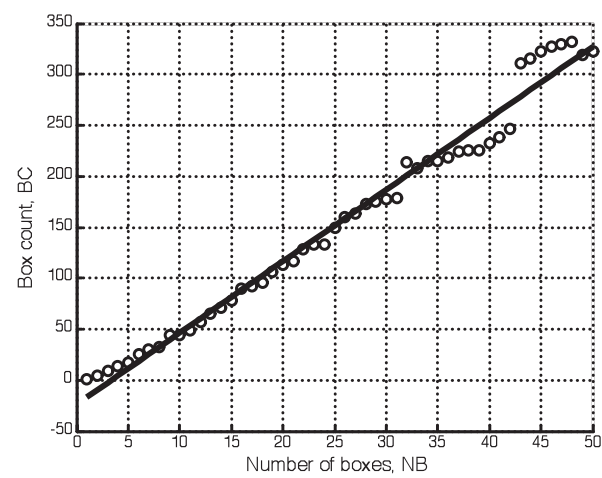

(1)

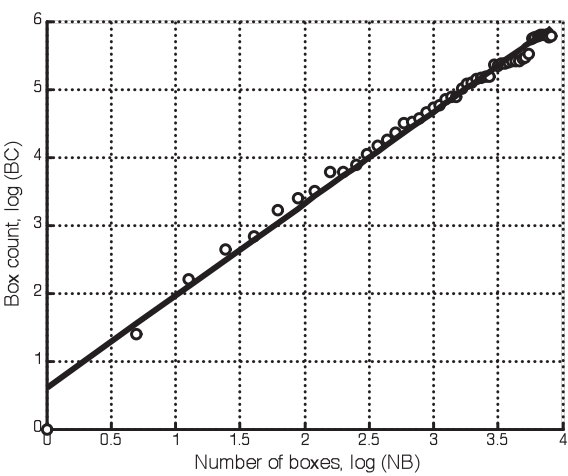

(2)

Figure 6e. (1) Box count (BC) vs. number of boxes (NB). (2) $\log (\mathrm{BC})$ vs. $\log (\mathrm{NB})$. 


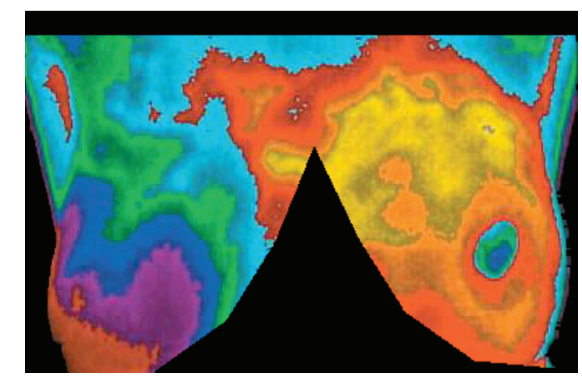

Figure 7a. Malignant case 2 (M2).
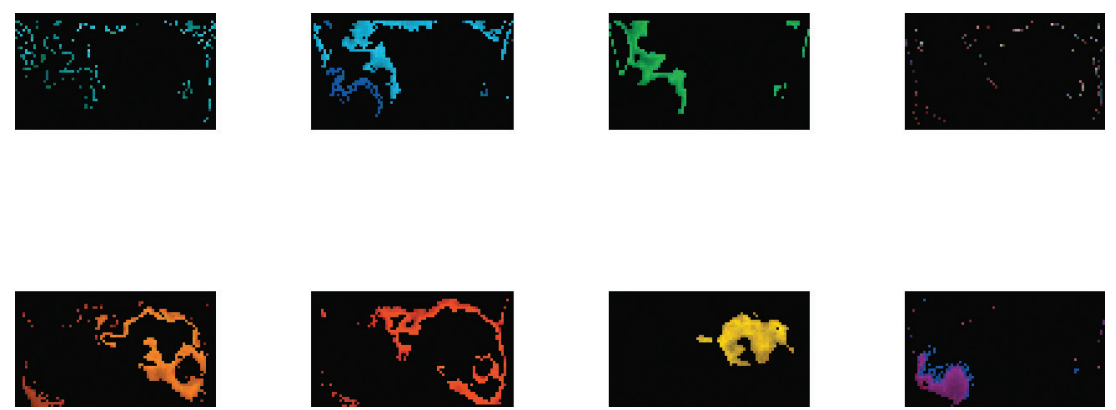

Figure7b. Segmentation of Figure 7a by fuzzy c-mean.

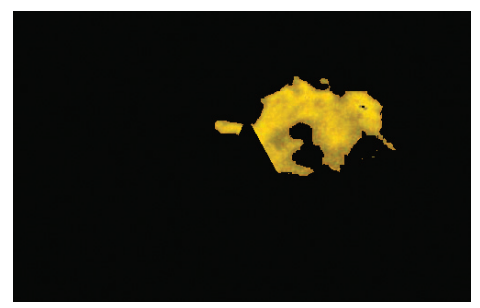

Figure 7c. The first hottest regions.

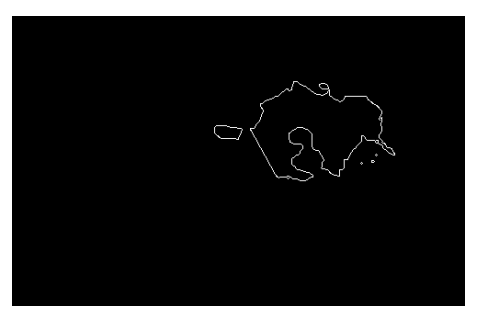

Figure 7d. Boundary contours of the first hottest regions. 


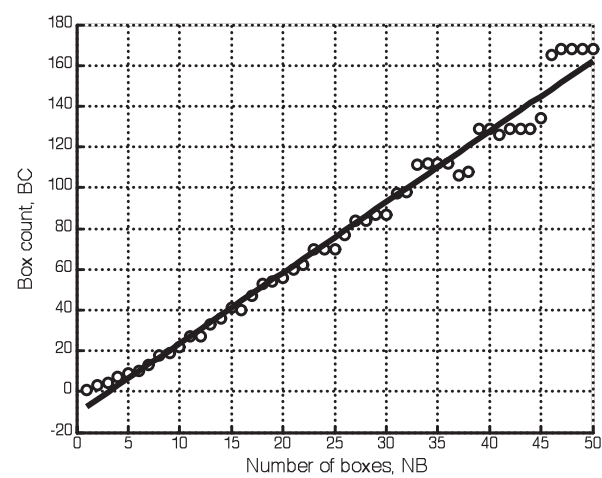

(1)

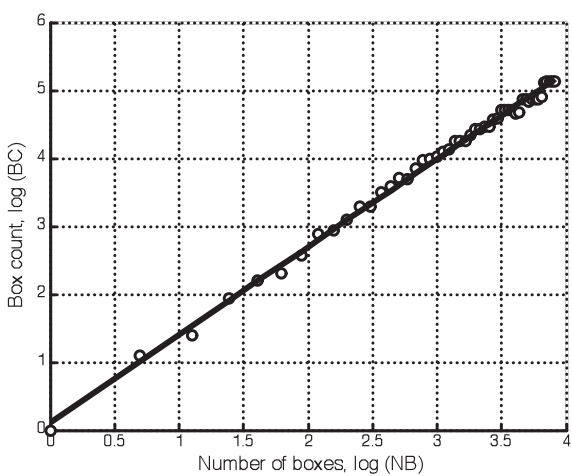

(2)

Figure 7e. (1) Box count (BC) vs. number of boxes (NB). (2) $\log (\mathrm{BC})$ vs. $\log (\mathrm{NB})$.

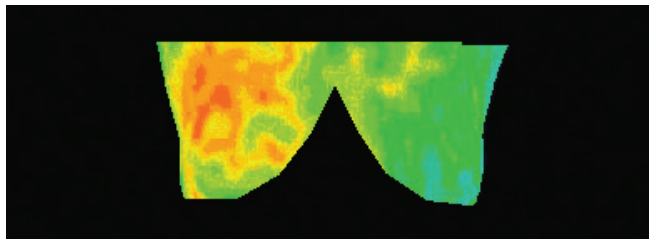

Figure 8a. Malignant case 3 (M3).
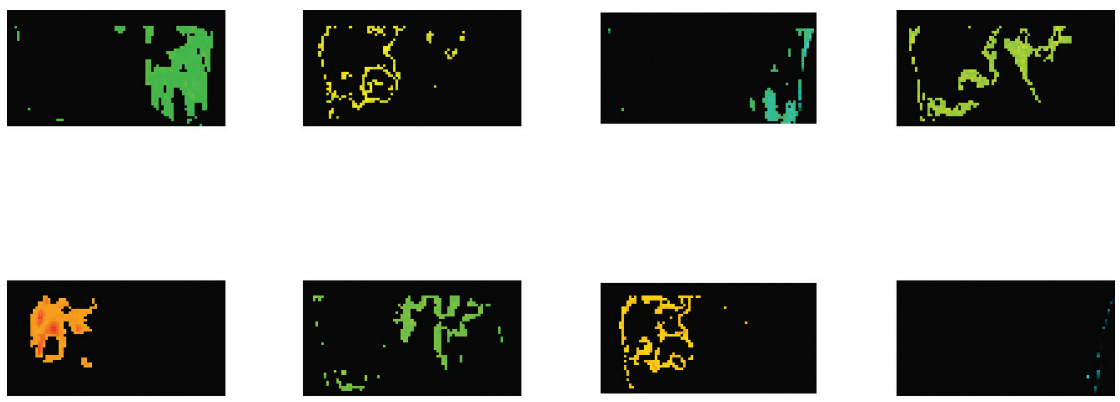

Figure 8b. Segmentation of Figure 8a by fuzzy c-mean.

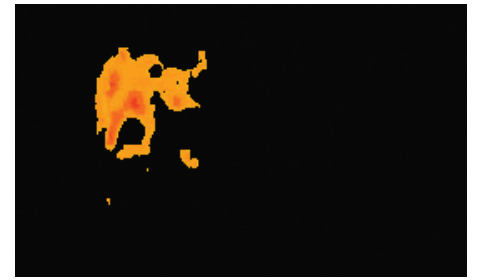

Figure 8c. The first hottest regions. 


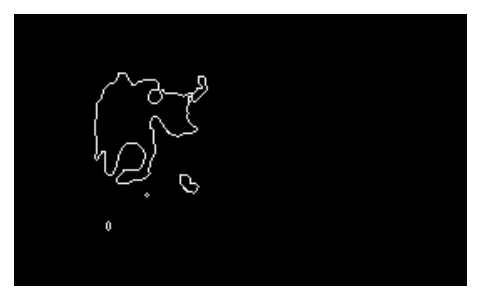

Figure 8d. Boundary contours of the first hottest regions.

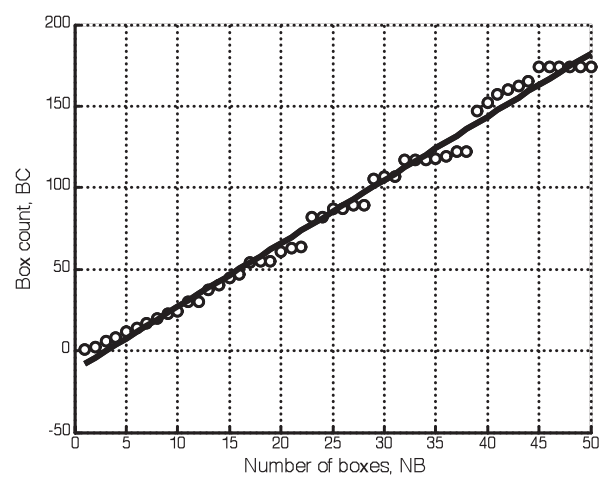

(1)

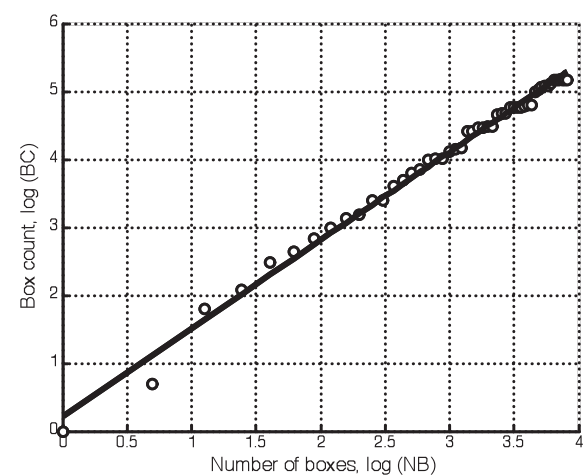

(2)

Figure 8e. (1) Box count (BC) vs. number of boxes (NB). (2) $\log (\mathrm{BC})$ vs. $\log (\mathrm{NB})$.

The FDs for eight benign and seven malignant cases were calculated as shown in Table 2, and the mean and standard deviation of calculated FDs are presented in Table 3. The FD results for benign cases are not significantly different from 1, the topological dimension of a line, while those for malignant cases are significantly greater.

Table 2. Calculated FDs for eight benign and seven malignant cases by BCM (B: Benign, M: Malignant)

$\begin{array}{ll}\text { B1 } & 0.9675 \\ \text { B2 } & .9911 \\ \text { B3 } & 1.1321 \\ \text { B4 } & 1.106 \\ \text { B5 } & 1.0382 \\ \text { B6 } & 0.9781 \\ \text { B7 } & 1.1218 \\ \text { B8 } & 1.0081 \\ \text { M1 } & 1.3538 \\ \text { M2 } & 1.2992 \\ \text { M3 } & 1.3479 \\ \text { M4 } & 1.2787 \\ \text { M5 } & 1.4001 \\ \text { M6 } & 1.331 \\ \text { M7 } & 1.2884\end{array}$


Table 3. Mean and standard deviation of calculated FDs for eight benign and seven malignant cases

\begin{tabular}{lcr}
\hline & Benign & Malignant \\
\hline Mean & 1.0429 & 1.3284 \\
Standard deviation & 0.0676 & 0.0430
\end{tabular}

Field implementation of this procedure is in progress in the Seyedo Shohada Hospital in Isfahan, Iran. Initial results have been very satisfactory, and many subjects appreciated the painless, non-invasive, and low-cost procedure. Results of this on-going study will be reported in another paper.

\section{CONCLUSION}

In this study, we analyzed thermal images of breast using fractal dimension to determine the possible difference between malignant and benign patterns. The present numerical experimental results verify the theoretical concepts and show a significant difference in FD between malignant and benign cases, with the FDs for benign cases close to 1 , while those for malignant cases significantly greater. This suggests that fractal analysis may potentially improve the reliability of thermography in breast tumor detection.

Fractal dimension is very sensitive to algorithm that segment images. For future work with a vast library of IR images at hand, validation of fuzzy c-means to extract pathologically relevant structures can be demonstrated through comparing the regions segmented by fuzzy c-means with the regions found by other modalities such as mammogram.

\section{REFERENCES}

[1] Jones, B. F., A reappraisal of the use of infrared thermal image analysis in Medicine, IEEE Transaction on Medical Imaging, 17(6), 1998, pp. 1019-1027.

[2] $\mathrm{Ng}$, E.Y. K., A review of thermography as promising non-invasive detection modality for breast tumor, International Journal of Thermal Sciences, 48(5), 2008, pp. 849-855.

[3] Diakides, N., Bronzino, J.D., Medical Infrared Imaging, CRC Press, Taylor \& Francis Group, 2008

[4] Qi, H., Kuruganti, P., Liu, Z. Early detection of breast cancer using thermal texture maps, Proceeding In IEEE International Symposium on Biomedical Imaging, Macro to Nano, 2002, pp: 309-312.

[5] $\mathrm{Ng}$, E.Y. K, Kee, E. C., Advanced integrated technique in breast cancer thermography, Journal of Medical Engineering \& Technology, 32(2), 2007, pp. 103-114.

[6] Ng, E.Y.K. Ng, G.J.L. Kaw, W.M. Chang, Analysis of IR thermal imager for mass blind fever screening, Microvascular Research, 68(2), 2004, pp. 104-109.

[7] Foster, K.R., Thermographic Detection of Breast Cancer, IEEE Engineering in medicine and biology Magazine, 17(6), Nov-Dec. 1998, pp. 10-14.

[8] Keyserlingk, P.D., Ahlgren, J.R, Yu, E., and Belliveau, N., Infrared Imaging of Breast: Initial Reappraisal using High- Resolution Digital Technology in 100 Successive Cases of Stage I and II Breast Cancer, The Breast Journal, 4(4), July- Aug. 1998, pp. 241-251.

[9] Ahmed, E., Fractals and chaos in cancer models, International Journal of Theoretical Physics, 32(2), 2004, pp. 353-355. 
[10] Baish, J.W., Jain, R. K., Fractals and Cancer, American Association for Cancer Research _Cancer Research, 60, 2000 3683-3688.

[11 Gazit, Y., Baish J.W., Safabakhsh, N., Leuning M., Baxter, L.T., Jaim, R.K., Fractal Characteristics of Tumor Vascular Architecture During Tumor Growth and Regression, Microcirculation, 4(4), 1997 , pp. 395-402.

[12] NCI: www.cancer.gov/cancertopics/UnderstandingCancer/angiogenesis (last accessed July 2009)

[13] Gamagami, P., Indirect signs of breast cancer: Angiogenesis study, In Allas of Mammography, Cambridge, MA Blackwell Science, 1996, pp. 231-258.

[14] Guidi, AJ., Schnitt, S.J., Angiogenesis in preinvasive lesions the breast, The Breast J, 2(4), 1996, pp. 364-369.

[15] Alarcon, T., Byrne, H.M., Maini, P.K., A cellular automaton model for tumour growth in inhomogeneous environment. J. Theor. Biol., 225(2), 2003, pp. 257-274.

[16] Smiley, M.W., Levine, H.A., Numerical Simulation of Capillary Formation During The Onset of Tumor Angiogenesis, Proceedings of the $4^{\text {th }}$ International Conf. on Dynamical System and Differential Equations, Wilmington, NC, USA, 2002, pp. 817-826.

[17] Singh, Y., Tumor Angiogenesis: Clinical Implications, Nepal Journal of Neuroscience, 1(2), 2004, pp. 61-63.

[18] Pisano, E.D., Breast Imaging, Vol. 13 Breast Disease, 130 pp, ISBN : 978-1-58603-168-8, 2002, I O S Press, Incorporated, VA, USA.

[19] Scalerandi, M., Pescarmona, G.P., Delsanto, P.P., and Capogrosso Sansone B., Local interaction simulation approach for the response of the vascular system to metabolic changes of cell behavior, Phys. Rev. E. Stat. Nonlin. Soft Matter Phys., 2001, 63(1 Pt 1), 011901.

[20] Anbar, M., Hyperthermia of the cancerous breast: analysis of mechanism, Cancer Lett., 84(1), 1994, pp. 23-29.

[21] Anbar, M., Breast cancer. In: Quantitative Dynamic Telethermometry in Medical Diagnosis and Management, Ann Arbor, MI CRC Press, 1994, pp. 84-94.

[22] Lee, T.K., McLean, D.I., Atkins, M.S., Irregularity index, a new border irregularity measure for cutaneous melanocytic lesions, Med Image Anal, 7(1), Mar. 2003, pp. 47-64.

[23] Zheng, L., Chan A..K., An artificial intelligent algorithm for tumor detection in screening mammogram, IEEE Trans Med Image, 20(7), 2001, pp. 559-567.

[24] Guo, Q., Ruiz, V., Shao, J., Guo, F., A novel approach to mass abnormality detection in mammographic images, In proceeding of the IASTED International Conference on Biomedical Engineering, Innsbruck, Austria, February 2005, pp 180-185.

[25] Caldwell, C.B., Stapleton, S.J., Holdsworth, D.W., Jong, R.A., Weiser, W.J., Cooke, G., and Yaffe, M.J., Characterization of mammographic parenchymal pattern by fractal dimension, Phys Med Biol, 35(2), 1990, pp. 235-247.

[26] Byng, JW., Boyd, NF., Fishell, E., Jong, RA., and Yaffe, MJ., Automated analysis of mammographic densities, Phys Med Biol, 41, 1996, pp. 909-923.

[27] Gazit, Y., Berk, D.A., Leunig, M., Baxter, L.T., and Jain, R.K., Scale-invariant behavior and vascular network formation in normal and tumor tissue, Phys. Rev. Lett, 75(12), Sep. 1995, pp. 2428-2431.

[28] Grizzi, F., Russo, C., Colombo, P., Franceschini, B., Frezza, E., Cobos, E., and Chiriva-Internati, M., Quantitative evaluation and modeling of two-dimensional neovascular network complexity: the surface fractal dimension, BMC Cancer, 5(14), 2005, DOI: 10.1186/1471-2407-5-14.

[29] Liu, J.Z., Zhang, L. D., and Yue, G. H., Fractal Dimension in Human Cerebellum Measured by Magnetic Resonance Imaging, Biophys J., 85(6), 2003 December, pp. 4041-4046.

[30] Kuczynski, K., and Mikotajczak, Magnetic Resonance Image classification using Fractal Analysis, Information Technologies in Biomedicine, Springer Berlin, 2008.

[31] Lee,W. L.,Chen, Y.C., Chen, Y. Ch., Unsupervised Segmentation of Ultrasonic Liver Images by Multiresolution Fractal Feature Vector, Information Science, 175(3), oct 2005, pp. 177-195 
[32] Esgiar, A.N., and Chakravorty, P.K., Fractal Based Classification of Colon Cancer Tissue images, IEEE, $9^{\text {th }}$ International Symposium on Signal Processing and its Application, Sharjah, United Arab Emirates Feb 2007, pp. 1-4.

[33] Rangayyan, R. M., and Nguyen, T.M., Fractal Analysis of Contours of Breast Masses in Mammograms, Journal of Digital Imaging, 20(3), 2007, pp. 223-237.

[34] Mastsubara, T., Fujita, H., Kasai, S., Goto, M., Tani, Y., Hara, T., and Endo, T., Development of new schemes for detection and analysis of mammographic masses, Proceedings of the 1997 IASTED International Conference on Intelligent Information Systems (IIS97), Grand Bahama Island, Bahamas, December 1997, pp. 63-66.

[35] Homer, M.J., Mammographic Interpretation, A Practical Approach, McGraw-Hill, Boston, MA, $2^{\text {nd }}$ edition, 1997.

[36] Reston, V.A., American College of Radiology, Illustrated Breast Imaging Reporting and Data System (BI-RADSTM), $3^{\text {rd }}$ edition, 1998.

[37] Mandelbrot, B.B., The fractal geometry of nature, 1983, San Francisco, CA, WH Freeman.

[38] Peitgen, H.O. Jurgens, H., and Saupe, D., Chaos and Fractal, New Frontiers of Science, 2004, New York, NY, Springer.

[39] Liu, S.H., Formation and anomalous properties of fractals, IEEE Eng Med Biol Mag, 11(2), 1992, pp. 28-39.

[40] Deering, W., and West,B.J., Fractal physiology, IEEE Eng Med Biol Mag, 11(2), June 1992, pp. 4046.

[41] Schepers, H.E., Van Beek, J.H.G.M., and Bassingthwaighte, J.B., Four methods to estimate the fractal dimension from self affine signals, IEEE Eng Med Biol Mag, 11(2), June 1992, pp. 57-64.

[42] Fortin, C.S., Kumaresan, R., Ohley, W.J., and Hoefer, S., Fractal dimension in the analysis of medical images, IEEE Eng Med Biol Mag, 11(2), June1992, pp. 65-71.

[43] Goldberger, A.L., Rigney, D.R.,and West, B.J., Chaos and fractals in human physiology, Scientific American, 262(2), 1990, pp. 42-49.

[44] AAT: http://aathermography.com (last accessed July 2009)

[45] MII: http://www.breastthermography.com/case_studies.htm (last accessed July 2009)

[46] ACCT: www.thermologyonline.org/Breast/breast_thermography_what.htm (last accessed July 2009)

[47] http://www.thermographyofiowa.com/casestudies.htm (last accessed July 2009)

[48] STImaging: http://www.stimaging.com.au/page2.html (last accessed July 2009)

[49] EtehadTavakol, M., Sadri, S., and Ng, E. Y-K., Application of K- and Fuzzy c-Means for Color Segmentation of Thermal Infrared Breast Images, Journal of Medical Systems, Vol. 34, No. 1, (2010), (DOI: $10.1007 / \mathrm{s} 10916-008-9213-1)$, pp. 35-42. 



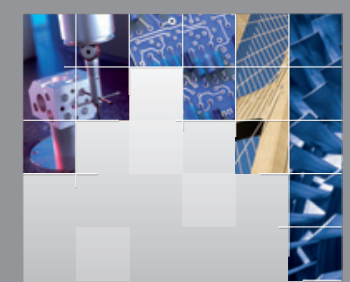

\section{Enfincering}
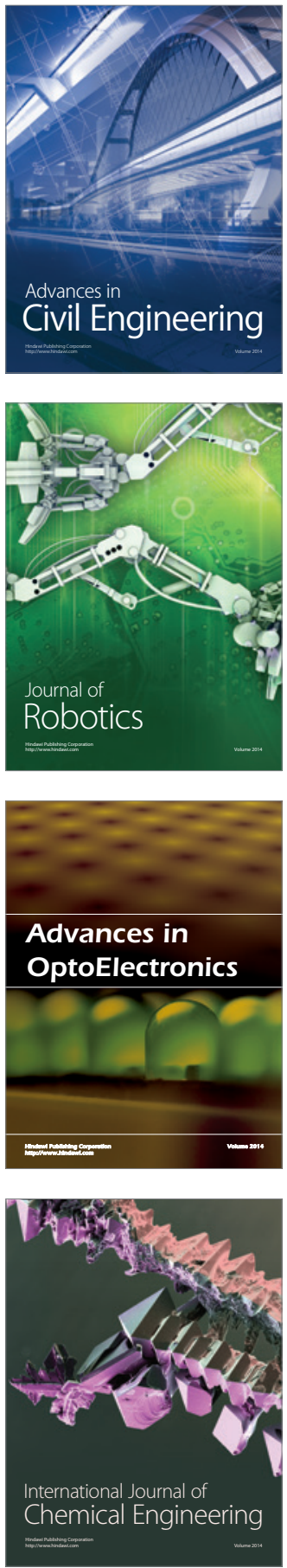

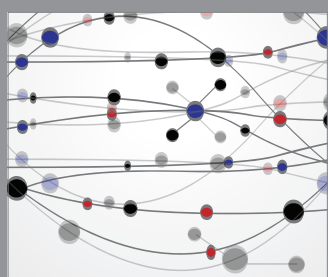

The Scientific World Journal

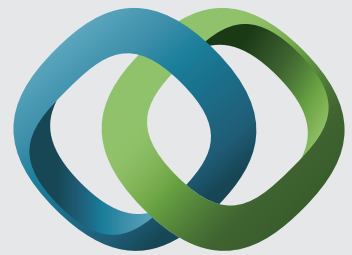

\section{Hindawi}

Submit your manuscripts at

http://www.hindawi.com
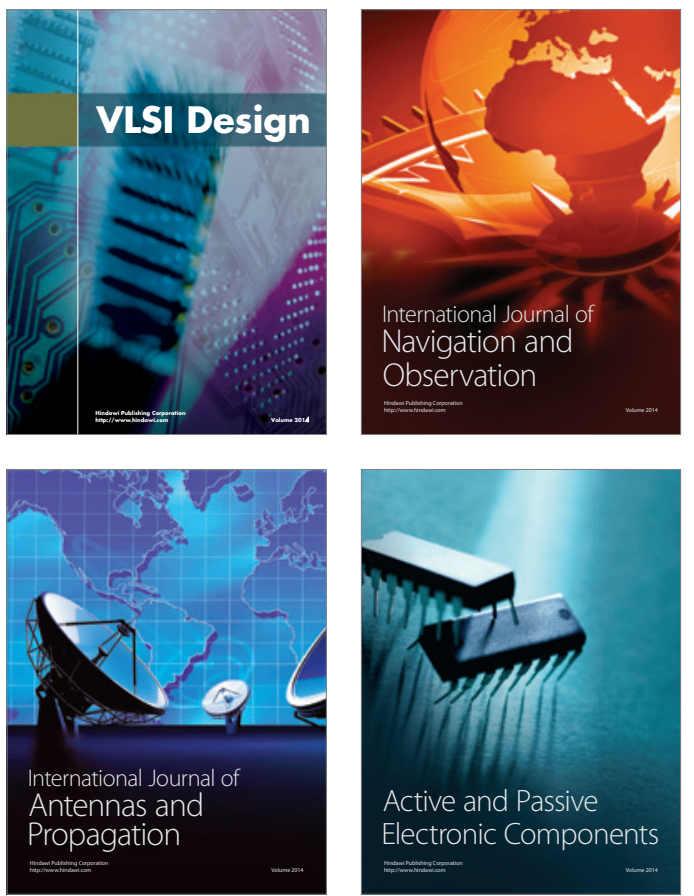
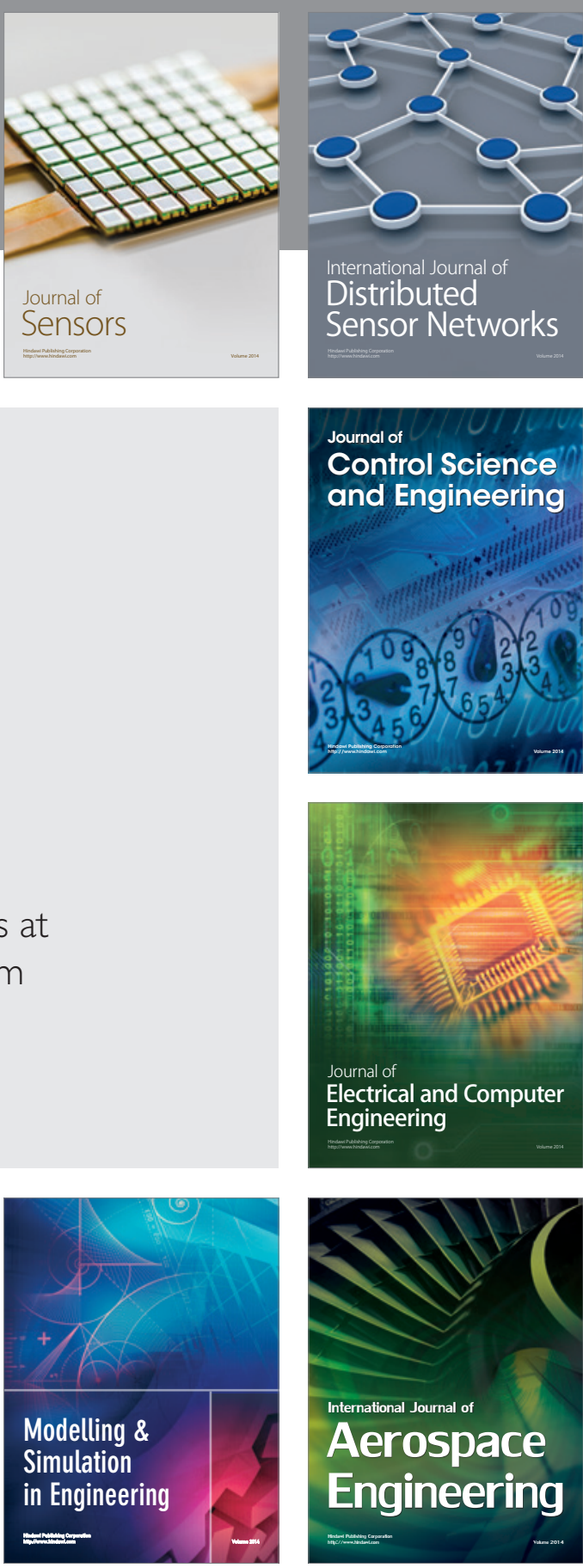

International Journal of

Distributed

Sensor Networks

Journal of

Control Science

and Engineering
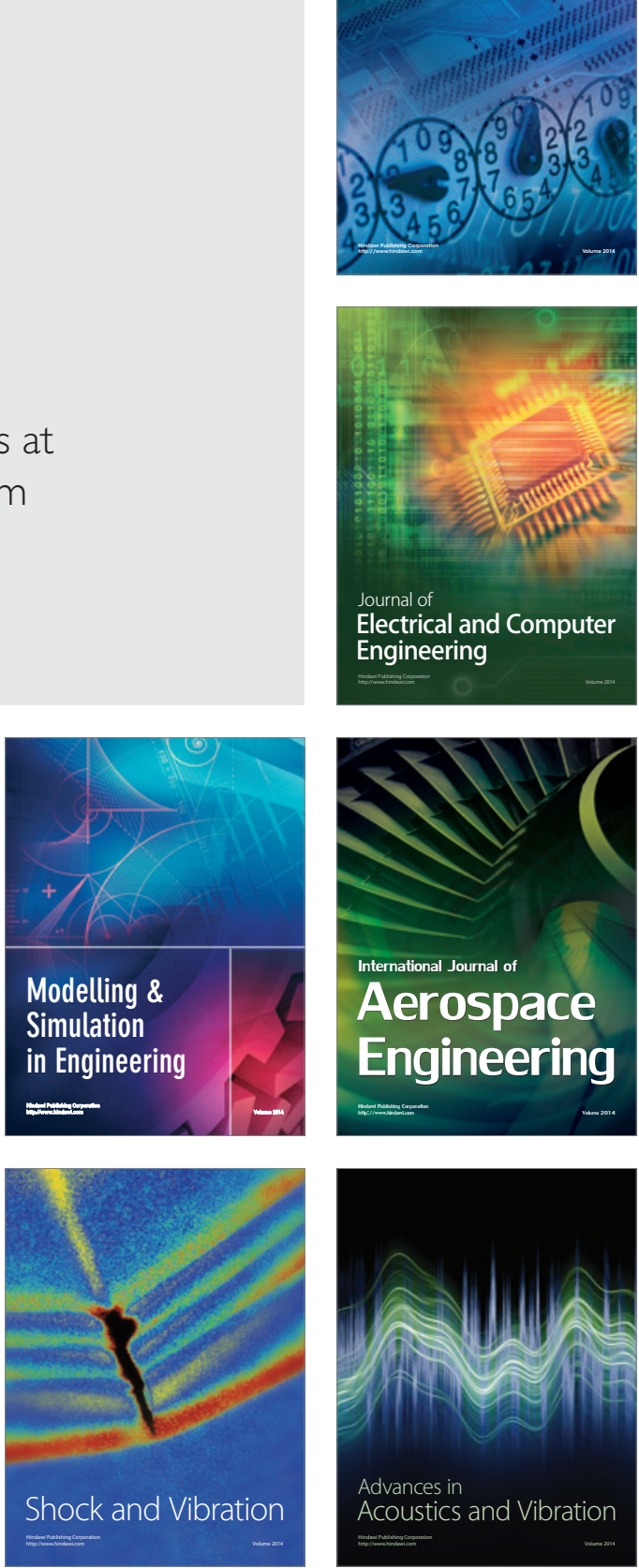